Tae Won Kim · Jae C. Choe

\title{
The effect of food availability on the semilunar courtship rhythm in the fiddler crab Uca lactea (de Haan) (Brachyura: Ocypodidae)
}

Published online: 2 September 2003

(C) Springer-Verlag 2003

\section{Behav Ecol Sociobiol (2003) 54:210-217}

Due to a technical error, Table 4 was reproduced incorrectly in the print version of this article.

The correct Table is given here:

Table 4 Male courtship in two semilunar cycles during the food manipulation test [initiation day and median day were counted from the day at spring tide (1st cycle: 6 June, 2nd cycle: 23 June, respectively)]

\begin{tabular}{|c|c|c|c|c|c|c|}
\hline & & \multicolumn{3}{|l|}{ Food treatment } & \multirow{2}{*}{$\begin{array}{l}\text { Test } \\
\text { statistic }\end{array}$} & \multirow[t]{2}{*}{$P$} \\
\hline & & Supplemented & Untreated & Deprived & & \\
\hline \multirow{7}{*}{$\begin{array}{l}\text { First } \\
\text { semilunar } \\
\text { cycle }\end{array}$} & \multicolumn{6}{|c|}{ Semidome building } \\
\hline & Initiation day & $\begin{array}{l}3.9( \pm 1.7) \\
n=10\end{array}$ & $\underset{n=12}{5.1}( \pm 1.4)$ & $\begin{array}{c}4.9( \pm 1.2) \\
n=7\end{array}$ & $H=2.15$ & 0.341 \\
\hline & \multirow[t]{2}{*}{ Median day } & $4.8( \pm 1.6)$ & $5.4( \pm 0.8)$ & $6.3( \pm 0.4)$ & $H=2.69$ & 0.260 \\
\hline & & $n=7$ & $n=8$ & $n=2$ & & \\
\hline & \multicolumn{6}{|l|}{ Waving } \\
\hline & Initiation day & $\begin{array}{l}4.8( \pm 1.5) \\
n=10\end{array}$ & $\begin{array}{c}5.5( \pm 1.8) \\
n=11\end{array}$ & $\underset{n=6}{5.0}( \pm 1.7)$ & $H=0.75$ & 0.686 \\
\hline & Median day & $\begin{array}{c}4.8 \\
n=6\end{array}( \pm 1.4)$ & $\begin{array}{r}5.7 \\
n=9\end{array}( \pm 1.2)$ & $\underset{n=3}{5.7}( \pm 0.8)$ & $H=1.14$ & 0.565 \\
\hline \multirow{6}{*}{$\begin{array}{l}\text { Second } \\
\text { semilunar } \\
\text { cycle }\end{array}$} & \multicolumn{6}{|c|}{ Semidome building } \\
\hline & Initiation day & $\begin{array}{l}0.4( \pm 2.7) \\
n=17\end{array}$ & $\begin{array}{l}3.1 \\
n=16\end{array}( \pm 1.8)$ & $\begin{array}{c}3.1 \\
n=8\end{array}( \pm 2.7)$ & $H=9.82$ & $0.007 *$ \\
\hline & Median day & $\begin{array}{l}1.7 \\
n=16\end{array}( \pm 2.2)$ & $\begin{array}{c}3.6( \pm 1.1) \\
n=8\end{array}$ & $\begin{array}{l}3.8 \\
n=3\end{array}( \pm 1.9)$ & $H=6.64$ & 0.03 \\
\hline & \multicolumn{6}{|l|}{ Waving } \\
\hline & Initiation day & $\begin{array}{c}0.4( \pm 2.0) \\
n=17\end{array}$ & $\begin{array}{l}3.4( \pm 0.7) \\
n=18\end{array}$ & $\begin{array}{l}3.0 \\
n=12\end{array}( \pm 1.5)$ & $H=16.01$ & $<0.0005^{*}$ \\
\hline & Median day & $\begin{array}{l}2.6 \\
n=15\end{array}( \pm 1.7)$ & $\begin{array}{c}4.2( \pm 0.3) \\
n=12\end{array}$ & $\underset{n=6}{4.1}( \pm 0.9)$ & $H=12.05$ & $0.004 *$ \\
\hline \multicolumn{2}{|c|}{ Semidome building cycle } & $\begin{array}{l}12.5( \pm 3.1) \\
n=8\end{array}$ & $\begin{array}{l}15.2( \pm 2.3) \\
n=10\end{array}$ & $\mathrm{a}$ & $t=-2.12$ & 0.05 \\
\hline \multicolumn{2}{|c|}{ Waving cycle } & $\begin{array}{l}12.5( \pm 1.6) \\
n=8\end{array}$ & $\begin{array}{l}14.9( \pm 1.9) \\
n=10\end{array}$ & $\begin{array}{l}14.7( \pm 2.3) \\
n=3\end{array}$ & $H=5.97$ & 0.05 \\
\hline
\end{tabular}

$* P<0.05$ sequential Bonferroni correction, $n=8$ tests.

${ }^{a}$ No males built semidomes in all two cycles.

The online version of the original article can be found at http:// dx.doi.org/10.1007/s00265-003-0614-3

T. W. Kim · J. C. Choe (

Laboratory of Behavior and Ecology, Seoul National University,

School of Biological Sciences, 151-742 Seoul, South Korea

e-mail: jcchoe@snu.ac.kr

Tel.: +82-2-8808158

Fax: $+82-2-8827195$ 